\title{
High-fidelity optical quantum gates based on type-II double quantum dots in a
} nanowire

Taherkhani, Masoomeh; Willatzen, Morten; Denning, Emil Vosmar; Protsenko, Igor E.; Gregersen, Niels

Published in:

Physical Review B (Condensed Matter and Materials Physics)

Link to article, DOI:

10.1103/PhysRevB.99.165305

Publication date:

2019

Document Version

Publisher's PDF, also known as Version of record

Link back to DTU Orbit

Citation (APA):

Taherkhani, M., Willatzen, M., Denning, E. V., Protsenko, I. E., \& Gregersen, N. (2019). High-fidelity optical quantum gates based on type-Il double quantum dots in a nanowire. Physical Review B (Condensed Matter and Materials Physics), 99(16), [165305 ]. https://doi.org/10.1103/PhysRevB.99.165305

\section{General rights}

Copyright and moral rights for the publications made accessible in the public portal are retained by the authors and/or other copyright owners and it is a condition of accessing publications that users recognise and abide by the legal requirements associated with these rights.

- Users may download and print one copy of any publication from the public portal for the purpose of private study or research.

- You may not further distribute the material or use it for any profit-making activity or commercial gain

- You may freely distribute the URL identifying the publication in the public portal 


\title{
High-fidelity optical quantum gates based on type-II double quantum dots in a nanowire
}

\author{
Masoomeh Taherkhani, ${ }^{1}$ Morten Willatzen, ${ }^{2,1}$ Emil V. Denning, ${ }^{1}$ Igor E. Protsenko, ${ }^{3}$ and Niels Gregersen ${ }^{1}$ \\ ${ }^{1}$ Department of Photonics Engineering, Technical University of Denmark, Ørsteds Plads, Building 343, 2800 Kongens Lyngby, Denmark \\ ${ }^{2}$ Beijing Institute of Nanoenergy and Nanosystems, Chinese Academy of Sciences, Haidian District, Beijing 100083, China \\ ${ }^{3}$ Lebedev Physical Institute of RAS, Leninsky Prospekt 53, Moscow 119991, Russia
}

(Received 18 April 2018; revised manuscript received 5 March 2019; published 10 April 2019)

\begin{abstract}
We propose an optical gating scheme for quantum computing based on crystal-phase type II double quantum dots in an InP nanowire. The qubit is encoded on the electron spin and the gate operations are performed using stimulated Raman adiabatic passage (STIRAP), using the orbital degree of freedom in double quantum dots to form an auxiliary ground state. Successful STIRAP gating processes require an efficient coupling of both qubit ground states of the double quantum dot to the gating auxiliary ground state, and we demonstrate that this can be achieved using a charged exciton state. Crucially, by using type II dots, the hole is localized between the two spatially separated electrons in the charged exciton complex, thereby efficiently coupling the electron groundstate orbitals. By taking advantage of the high-fidelity state transfer by means of STIRAP in type II double quantum dots, we propose a protocol for coherently manipulating the spin-orbital quantum state of confined electrons in a quantum dot chain of an InP nanowire. We subsequently exploit the protocol to realize single- and two-qubit gates with fidelity above 0.99 .
\end{abstract}

DOI: 10.1103/PhysRevB.99.165305

\section{INTRODUCTION}

Qubits encoded in electrons in semiconductor quantum dots (QDs) have been widely investigated as potential building blocks for quantum computers [1-3]. Since charge and spin noise processes are slow relative to the radiative decay rate, close to dephasing-free qubit operations are possible in ultraclean semiconductor QDs by working at high frequencies or through dynamical decoupling [4-6]. This property combined with remarkable progress in initialization, control and readout of the semiconductor qubits has made QDs promising for quantum information processing [1,7-9]. The magnetic interaction between spin qubits is generally too weak for fast two-qubit operations, and for this reason the spin qubit usually has to, at least briefly, adopt a charge character during the gate operation [10]. A charge qubit, where the information is stored on the location of an electron in a few-QD structure, can exploit strong Coulomb interaction, and thus fast gate operation well into the gigahertz range can be achieved $[10,11]$. The combination of the spin and charge manipulations in semiconductor few-QD structures to take advantage of the long memory times associated with spin states and at the same time manipulation of charge states has made them a popular platform for quantum computation [12].

Troiani et al. [13] proposed to use the spin of an excess electron in a type I double QD (DQD) as a qubit realization combined with a charge encoding for fast gating operations. In their laterally arranged DQD array, qubits are manipulated by means of the all-optical STIRAP technique. STIRAP is an efficient method for transferring populations adiabatically between two discrete quantum states by coupling them using two radiation fields via an intermediate state which is usually a radiatively decaying state $[14,15]$. When the adiabatic condition is fully satisfied, the evolution of the system is robust with respect to control parameter variations such as the laser intensity, the pulse timing and the pulse shape, and this property has made STIRAP a popular tool in optical quantum information processing $[16,17]$. The coupling can be optically turned on and off, so that single- and two-qubit operations can be selectively chosen as required in a scalable quantum computation scheme [18]. However, the charged exciton state of the type I DQD used as the intermediate state in the proposed scheme in Ref. [13] does not allow for good overlap between the hole and both electron wave functions simultaneously, however this simultaneous coupling is essential for implementing high-fidelity quantum gates by means of the STIRAP gating technique.

On the other hand, the type II band structure allows for the possibility of good overlap between electrons of neighboring QDs with a common hole state. It was recently shown that the otherwise weak transition oscillator strength of the exciton of a crystal-phase type II QD in an InP nanowire can be increased [19] by implementing a DQD structure leading to an improved overlap between the electron and hole parts confined inside the QD and the barrier, respectively. Additional advantages of DQDs in nanowires include the possibility for implementing multiple local metallic contacts and electrostatic gates on top, beneath, and next to the wire, which can be coupled to measuring apparatus or to adjacent DQD nanowires [20,21]. The laser beam used in STIRAP can be focused on a specific nanowire site which guarantees individual addressability of the quantum gates [16,22]. However, global-manipulation schemes are also possible [23].

It was suggested in Ref. [13] that the type II QDs may be more suitable than type I QDs for qubit manipulations by STIRAP, however this was to our knowledge never investigated. 
In this paper, we show that type II QDs do indeed perform significantly better than type I QDs for quantum gating using STIRAP. Whereas many works present phenomenological optical gating models with parameters unrelated to a realistic geometrical system $[3,10]$, we propose a specific type II InP nanowire system and employ realistic parameters computed for this specific geometry. We first introduce a DQD encoding a single qubit with a charged exciton configuration featuring a mixed hole part acting as an intermediate state for the STIRAP process. We show that this mixed hole configuration allows for efficient coupling of the full two-dimensional spin qubit space localized in one QD of the DQD structure to an electron state in the other QD as required for single-qubit gate operations. We then introduce a specific system consisting of two neighboring DQDs in the nanowire each encoding one qubit. We show that the strong Coulomb interaction between the charges, which causes a significant shift of the STIRAP transition frequencies, can be exploited to efficiently perform conditional two-qubit CNOT operation on the two qubits. Importantly, we also show that the implementation is robust against decoherence posed by spin and charge fluctuations in the environment. This robustness is largely attributed to the flexibility of the scheme with respect to bandwidths and dynamical timescales ensured by using STIRAP for rapid control operations.

To evaluate the dipole moments, we introduce a full multiband formalism taking into account band-mixing effects. This formalism is compatible with methods such as $k \cdot p$ theory, empirical tight binding, and ab initio [24-26], which allow for accurate modeling of excitons in QDs by taking into account detailed electronic band structures. However, to obtain a numerically fast model suitable for design optimization, we perform our dipole moment calculations using a single-band model based on the envelope function and effective mass approximations. Additionally, in the type II DQD structure considered in this work, the electron and the hole are confined respectively by the band-edge potential profile inside the QDs and inside the barrier [19] and the Coulomb interaction is negligible in comparison to the potential profile confinement. For this reason, we have not considered the Coulomb interaction when calculating the charged exciton state. It was shown by Faria Jr. et al. [26] that strain and polarization fields do not have strong influence on the optical properties of type II InP crystal phase QDs, and these effects were for this reason neglected in their later work [27]. Accordingly, we neglect the influence of strain, piezoelectric effects and polarization fields.

The paper is organized as follows. In Sec. II, we discuss the implementation of the STIRAP scheme for DQDs in nanowires, and the details of our theoretical model are summarized in Sec. III. The operation of the quantum gates based on the proposed DQD geometries by means of STIRAP is presented in Sec. IV. In Sec. V, we present the calculation of the quantum gates fidelities by solving the master equation and taking into account the different dephasing rates. Section VI is a discussion on physical constraints of the process followed by a conclusion in Sec. VII. Details of the calculation of the dipole matrix elements and of our initialization scheme are presented in Appendices A and B, respectively. (a)

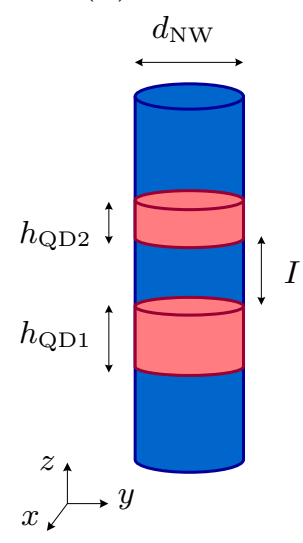

(b)

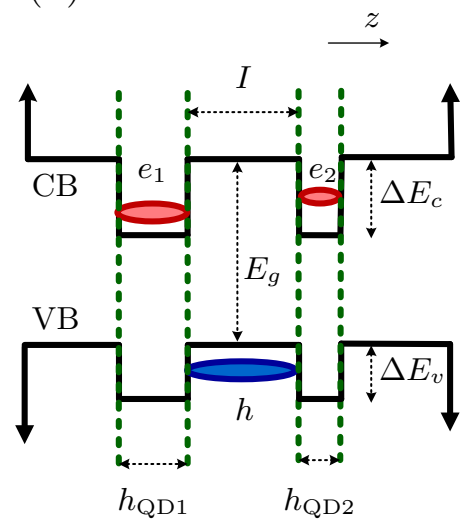

FIG. 1. (a) A DQD in a nanowire. (b) The conduction and valence band potential energy profiles along the $z$ axis for the type II DQD with electron (red) and hole (blue) wave functions schematically illustrated.

\section{PHYSICAL IMPLEMENTATION}

We consider crystal phase DQDs implemented in a rotationally symmetric InP nanowire shown schematically in Fig. 1(a) as a platform for our optical quantum gating scheme. The QDs are formed by a transition in the crystallographic lattice of the InP material between the zinc blende (ZB) and wurtzite (WZ) crystal phases during the fabrication process [24]. Within each DQD, we label the lower (upper) QD by $\mathrm{QD}_{1}\left(\mathrm{QD}_{2}\right)$. In these structures, the single-particle electron states reside inside the QDs as shown in Fig. 1(b), where the QD heights are chosen unequal, $h_{\mathrm{QD} 1} \neq h_{\mathrm{QD} 2}$, to separate the energy levels associated with the two electronic orbitals. Furthermore, an in-plane magnetic field splits the electron spin states. The spatial overlap of the electron ground states of the individual QDs is chosen to be negligible such that tunneling between electron states during gate operations is suppressed. Most single-particle hole states are predominantly localized in the outer nanowire regions surrounding the DQD. However, there are a few single-particle hole states which are mainly localized inside the barrier region between two QDs, and Fig. 1(b) shows the lowest energy hole state confined inside the barrier in the valence band. We are particularly interested in this hole state inside the barrier since the corresponding exciton [19] has a considerable dipole coupling to both electronic ground-state orbitals. Furthermore, due to the small real-space overlap of the hole wave functions, phonon decay processes from the ground-state exciton with hole part inside the barrier to the lower energy excitons with the hole part outside the barrier can be neglected [28].

In the STIRAP scheme for a three-level system, a pump pulse links the initial and intermediate state and a Stokes pulse links the intermediate and the final state. By applying the Stokes pulse before the pump pulse and by maintaining adiabatic evolution conditions, a so-called dark state is formed through which the population is transferred between ground states without populating the radiatively decaying intermediate state [15]. Figure 2 shows the STIRAP scheme between two electron states $\left|e_{1}\right\rangle$ and $\left|e_{2}\right\rangle$ of a DQD in a 

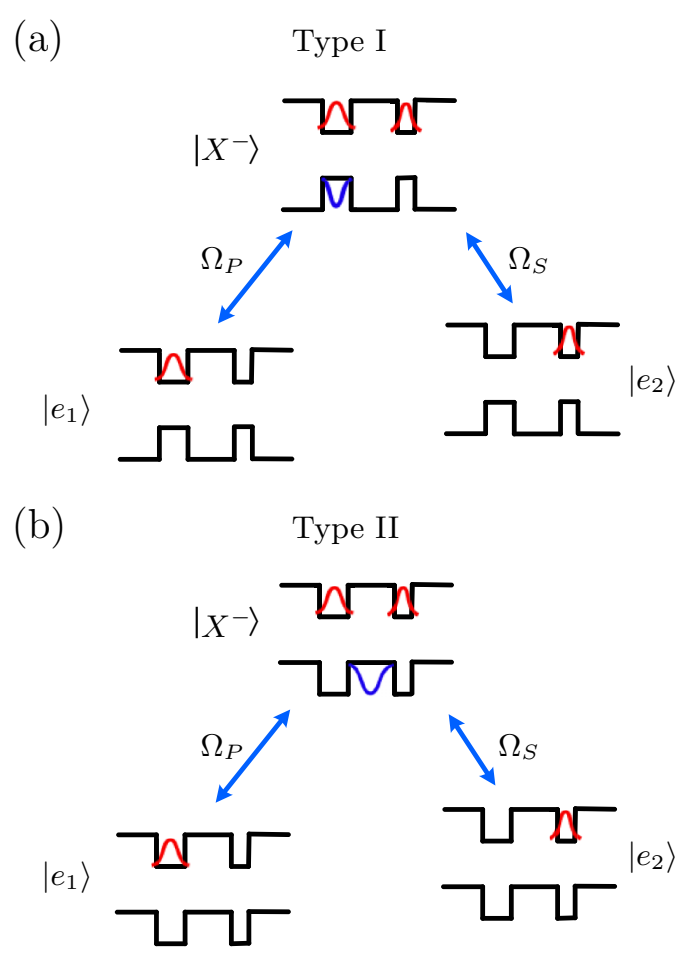

FIG. 2. Three-level STIRAP scheme in (a) type I and (b) type II DQDs.

type I and in a type II configuration. The interconnecting state in both cases is a charged exciton state $\left|X^{-}\right\rangle$, i.e., an electron-hole complex consisting of one electron in each QD and a single hole. Initially, the entire population is in the single-electron ground state $\left|e_{1}\right\rangle$ of $\mathrm{QD}_{1}$. The pump pulse induces the transition between the states $\left|e_{1}\right\rangle$ and $\left|X^{-}\right\rangle$, and the Stokes pulse enables the transition between the states $\left|X^{-}\right\rangle$ and $\left|e_{2}\right\rangle$, where $\left|e_{2}\right\rangle$ is the single-electron ground state of $\mathrm{QD}_{2}$. The coupling strengths of the pump $P$ and Stokes $S$ transitions are defined by the Rabi frequencies $\Omega_{P}(t)=d_{P} E_{P}(t) / \hbar$ and $\Omega_{S}(t)=d_{S} E_{S}(t) / \hbar$ with transition dipole moments $d_{P, S}$ and the electric fields of the lasers $E_{P, S}(t)$.

As illustrated in the Fig. 2(a), while the overlap between the hole and electron wave functions in the left dot is large in the type I configuration, the overlap between the electron in the right dot and the hole in the left dot is very small. Figure 3(a) shows the calculated $d_{P}$ and $d_{S}$ in a type I GaAs/AlGaAs DQD in a nanowire as a function of $I$ the distance between the QDs [cf. Fig. 1(a)]. Details about calculation of the dipole moments are presented in Appendix A and the material parameters used in the calculation are listed in Table I. As we observe in this figure, the dipole moment $d_{P}$ which is proportional to the overlap between $\left|e_{1}\right\rangle$ and $\left|X^{-}\right\rangle$ is nearly zero and the intermediate state $\left|X^{-}\right\rangle$couples very weakly to $\left|e_{1}\right\rangle$. For this reason the STIRAP gating scheme in the type I DQD configuration is highly inefficient. On the other hand, as shown in Fig. 2(b) the hole in the type II configuration is localized in the barrier region between the QDs and thus overlaps with the electrons of both QDs. Fig. 3(b) shows the calculated $d_{P}$ and $d_{S}$ in a type II crystal-phase InP DQD in a nanowire as a function of the distance $I$ between the QDs. As we see in this figure, while the dipole moment $d_{S}$ is not as
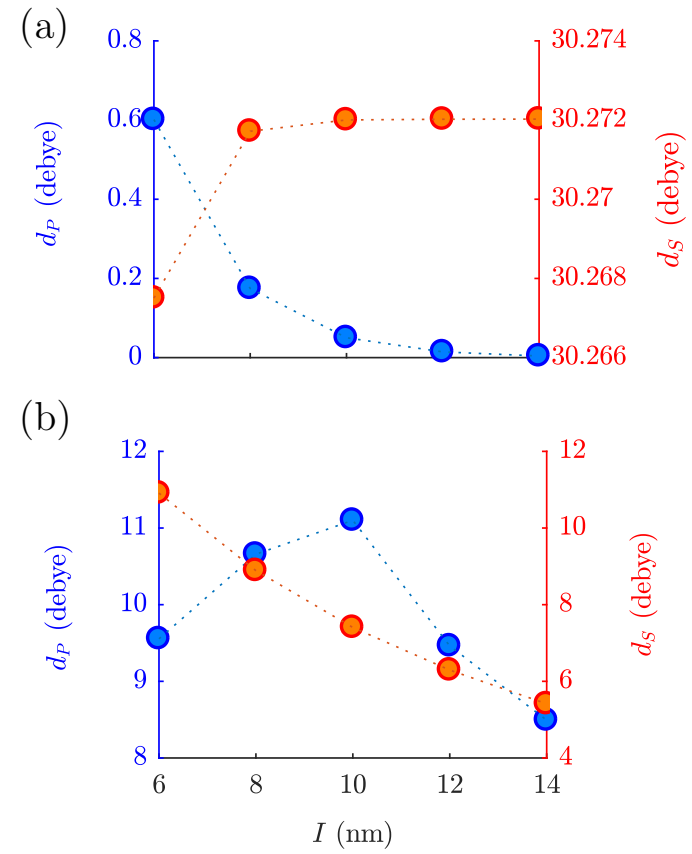

FIG. 3. Transition dipole moments $d_{P}$ and $d_{S}$ in (a) type I GaAs/AlGaAs DQD in a nanowire and (b) type II crystal-phase InP DQD in a nanowire. Note the different scales for the vertical axes. The geometrical parameters of the structure are $h_{\mathrm{QD} 1}=5 \mathrm{~nm}$, $h_{\mathrm{QD} 2}=3 \mathrm{~nm}, d_{\mathrm{QD}}=40 \mathrm{~nm}$, and $l_{\mathrm{NW}}=70 \mathrm{~nm}$.

large as that of the type I configuration, the magnitudes of the overlaps between the hole and the right electron proportional to $d_{P}$ and the hole and the left electron proportional to $d_{S}$ are similar. This property leads to Rabi frequencies $\Omega_{P}$ and $\Omega_{S}$ of similar magnitudes and represents a major asset of the type II configuration.

\section{THEORETICAL MODEL}

We initially consider a single electron in $\mathrm{QD}_{1}$ of the $\mathrm{DQD}$ structure. By applying a uniform magnetic field $B$ in the Voigt configuration along the $x$ axis (cf. Fig. 1), the electron spin states $\left|S_{x}= \pm \frac{1}{2}\right\rangle$ are split by $\mu_{B} g_{e} B$ by the Zeeman effect as shown in Fig. 4. Here, $\mu_{B}$ is the Bohr magneton and $g_{e}$ is the electron spin $g$ factor. The electron eigenstates used to encode the logical qubit of the DQD are then direct products of the

TABLE I. Material parameters.

\begin{tabular}{lcc}
\hline \hline Parameter & Value (InP) & Value $(\mathrm{GaAs})$ \\
\hline$\Delta E_{c}$ & $129 \mathrm{meV}$ & $400 \mathrm{meV}$ \\
$\Delta E_{v}$ & $65 \mathrm{meV}$ & $215 \mathrm{meV}$ \\
$E_{g}$ & $1.410 \mathrm{eV}$ & $1.424 \mathrm{eV}$ \\
$\epsilon$ & 12.5 & 12.9 \\
$2|M|^{2} / m_{0}[29]$ & $20 \mathrm{eV}$ & $28.8 \mathrm{eV}$ \\
$m_{e}^{*}$ & $0.068 m_{0}$ & $0.067 m_{0}$ \\
$m_{h}^{*}$ & $0.64 m_{0}$ & $0.38 m_{0}$ \\
$m_{0}$ & $9.1 \times 10^{-31} \mathrm{Kg}$ & \\
\hline \hline
\end{tabular}




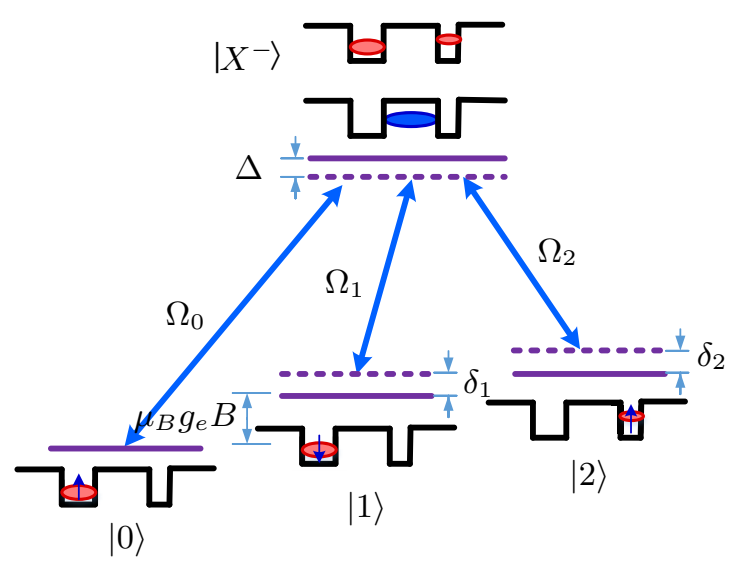

FIG. 4. The interaction scheme for manipulating the spin of an electron by STIRAP in the DQD structure. The qubit is defined by the ground states $|0\rangle$ and $|1\rangle$. The states $|2\rangle$ and $\left|X^{-}\right\rangle$are auxiliary states occupied only in the intermediate phase of the gating procedure. $\Omega_{0}$, $\Omega_{1}$, and $\Omega_{2}$ are the Rabi frequencies of the transitions, $\Delta$ and $\delta_{1,2}$ are, respectively, single- and two-photon detunings defined in the main text.

electron position and the spin degrees of freedom given by

$$
|0\rangle=\left|f_{e 1}\right\rangle \otimes\left|S_{x}=+\frac{1}{2}\right\rangle, \quad|1\rangle=\left|f_{e 1}\right\rangle \otimes\left|S_{x}=-\frac{1}{2}\right\rangle,
$$

where $\left|f_{e 1}\right\rangle$ is the ground-state electron envelope function in $\mathrm{QD}_{1}$.

In a system featuring three ground states coupled to an excited state, an arbitrary single-qubit rotation can be performed [30] using a STIRAP process. In the DQD configuration, we use the state $|2\rangle$ defined as

$$
|2\rangle=\left|f_{e 2}\right\rangle \otimes\left|S_{x}=+\frac{1}{2}\right\rangle,
$$

together with the states $|0\rangle$ and $|1\rangle$ defined in Eq. (1) as the three ground states of the single-qubit rotation scheme. $\left|f_{e 2}\right\rangle$ in Eq. (2) is the ground-state electron envelope function in $\mathrm{QD}_{2}$ of the DQD.

The excited state allowing optical coupling between the input space $\alpha|0\rangle+\beta|1\rangle$ and the state $|2\rangle$ is a negatively charged exciton state $\left|X^{-}\right\rangle$as shown in Fig. 4. Band mixing is pronounced in QDs since the effective $\mathbf{k}$ point is located substantially away from the $\Gamma$ point, such that the charged exciton's hole part is a mixed state. As it is shown in Appendix A, due to this mixing the charged exciton state couples with all the electron states $|0\rangle$ and $|1\rangle$ in $\mathrm{QD}_{1}$ and $|2\rangle$ in $\mathrm{QD}_{2}$. This possibility of efficient coupling of the intermediate state with all the electronic ground states is a requirement for single-qubit operations and represents a key asset of our gating scheme. The coupling strength of the charged exciton state to $|0\rangle,|1\rangle,|2\rangle$ depends on the bulk transition matrix elements $M$ and the overlap of the envelope functions, the detailed calculation of which is presented in Appendix A.

The differences between the laser frequencies and the corresponding transition frequencies are denoted by $\Delta_{0}, \Delta_{1}$ and $\Delta_{2}$. We then define the single-photon detuning as $\Delta \equiv$ $\Delta_{0}$ and two-photon detunings $\delta_{1} \equiv \Delta_{1}-\Delta_{0}, \delta_{2} \equiv \Delta_{2}-\Delta_{0}$ (cf. Fig. 4). The Hamiltonian describing the coupling of the four states $\left\{|0\rangle,|1\rangle,\left|X^{-}\right\rangle,|2\rangle\right\}$ using three coherent radiation

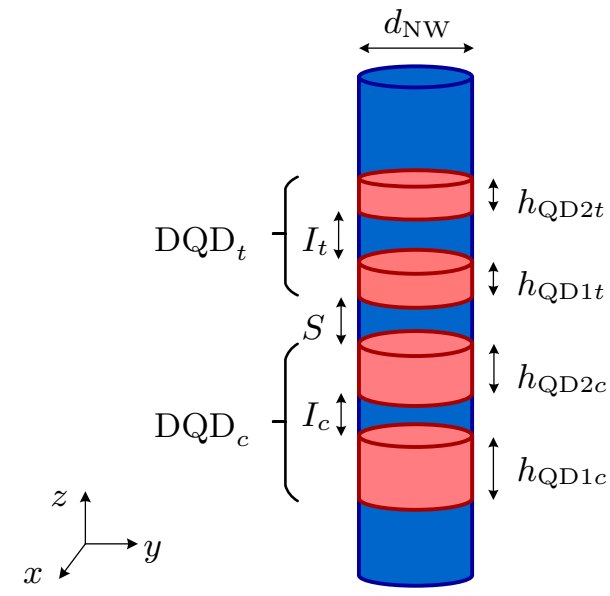

FIG. 5. Two DQDs in a nanowire. The geometrical parameters of $\mathrm{DQD}_{c}$ are $h_{\mathrm{QD} 1 c}=11 \mathrm{~nm}, h_{\mathrm{QD} 2 c}=10 \mathrm{~nm}, I_{c}=8 \mathrm{~nm}$, and the parameters of $\mathrm{DQD}_{t}$ are $h_{\mathrm{QD} 1 t}=5 \mathrm{~nm}, h_{\mathrm{QD} 2 t}=3 \mathrm{~nm}$, and $I_{t}=10 \mathrm{~nm}$. The nanowire diameter is $d_{\mathrm{NW}}=40 \mathrm{~nm}$.

fields within the rotating wave approximation is then given by [31]

$$
H=\frac{\hbar}{2}\left[\begin{array}{cccc}
0 & 0 & \Omega_{0} & 0 \\
0 & 2 \delta_{1} & \Omega_{1} & 0 \\
\Omega_{0}^{*} & \Omega_{1}^{*} & 2 \Delta & \Omega_{2}^{*} \\
0 & 0 & \Omega_{2} & 2 \delta_{2}
\end{array}\right] .
$$

The STIRAP transfer efficiency, which is the probability of successful population transfer from the initial to the target state, depends differently on $\delta_{1}, \delta_{2}$, and $\Delta$ : STIRAP is sensitive to the magnitude of the two-photon detunings $\delta_{1}$ and $\delta_{2}$ as the formation of the dark state requires two-photon resonances $\delta_{1}=\delta_{2}=0$. On the other hand, the formation of the dark state is not prevented by a nonzero single-photon detuning $\Delta$ [14].

In a realistic implementation of the protocol, the system will be subject to the spontaneous emission from the charged exciton, spin dephasing due to electron-nucleus interactions and pure dephasing of the charged exciton due to fluctuations in the local charge environment. To take these processes into account, we calculate the time evolution by solving the master equation [32]

$$
\begin{aligned}
\frac{d}{d t} \rho(t)= & -\frac{i}{\hbar}[H(t), \rho(t)]+\gamma_{\mathrm{PD}} \mathcal{L}\left(\left|X^{-}\right\rangle\left\langle X^{-}\right|\right) \\
& +\sum_{k=0,1,2}\left(\gamma_{\mathrm{SE}} \mathcal{L}\left(|k\rangle\left\langle X^{-}\right|\right)+\gamma_{\mathrm{SD}} \mathcal{L}(|k\rangle\langle k|)\right),
\end{aligned}
$$

where $\rho$ is the density matrix operator, $H$ is the isolated (closed) system Hamiltonian in Eq. (3), $\mathcal{L}$ is the Lindblad superoperator defined by $\mathcal{L}(x)=x \rho(t) x^{\dagger}-\frac{1}{2}\left(x^{\dagger} x \rho(t)+\right.$ $\left.\rho(t) x^{\dagger} x\right), \gamma_{\mathrm{PD}}$ is the pure dephasing rate of the charged exciton, $\gamma_{\mathrm{SE}}$ is the spontaneous emission rate from the charged exciton $\left|X^{-}\right\rangle$into the ground states $|0\rangle,|1\rangle$, and $|2\rangle$ and $\gamma_{\mathrm{SD}}$ is the ground-state spin dephasing rate.

\section{OPTICAL QUANTUM GATING}

In the following, we consider a nanowire featuring multiple DQDs as illustrated in Fig. 5 with each DQD encoding one 
logical qubit. The nanowire is illuminated from above such that the laser light propagates along the nanowire $z$ axis with a polarization vector $\mathbf{e}$ oriented in the plane perpendicular to the nanowire axis. Each DQD can be manipulated individually using STIRAP in a global-manipulation scheme [23] by choosing different geometrical parameters (QD thicknesses) for each DQD such that the STIRAP pulse frequencies are only resonant with one specific DQD. In the following, the pump pulses $E_{0}(t)=E_{P}(t) \cos \chi$ and $E_{1}(t)=$ $E_{P}(t) \sin \chi \exp (i \eta)$ couple $|0\rangle$ and $|1\rangle$ to $\left|X^{-}\right\rangle$, respectively. Here, $E_{P}(t)=E_{P} \exp (-t / \tau)^{2}$ and $\chi$ and $\eta$ are phase factors defined by the desired gate operation. The Stokes pulse $E_{2}(t)=E_{2} \exp (-(t+\Delta T) / \tau)^{2}$ couples $|2\rangle$ to $\left|X^{-}\right\rangle . E_{P}(t)$ and $E_{2}(t)$ are Gaussian shaped laser pulse envelopes with a time separation $\Delta T$ between the maxima of the pulses and a pulse width $\tau / \sqrt{2}$.

\section{A. Single-qubit gate}

To perform single-qubit gate operations, we exploit the possibility of coupling both spin ground states $|0\rangle$ and $|1\rangle$ to the auxiliary state $|2\rangle$ via the intermediate state $\left|X^{-}\right\rangle$, which is possible thanks to the mixed hole state of the charged exciton as discussed in Sec. III. This allows us to employ a standard STIRAP-based single-qubit rotation scheme [30] for the manipulations.

The single-qubit rotation takes place as follows. Initially the system is in the state $|i\rangle=\alpha|0\rangle+\beta|1\rangle$. The DQD structure is subject to the first STIRAP process followed by a second reversed STIRAP process. The phase factors $\chi$ and $\eta$ are the same in the both STIRAPs. In the second STIRAP two pump pulses arrive before the Stokes pulse and the $\Omega_{2}$ is phase shifted with respect to the $\Omega_{2}$ in the first STIRAP by a phase $\zeta$. After the two STIRAP processes, the initial state vector $|i\rangle$ is rotated by an angle $\zeta$ around the axis $\mathbf{n}=$ $(\sin 2 \chi \cos \eta, \sin 2 \chi \sin \eta, \cos 2 \chi)$ leading to a final state [30]

$$
|f\rangle=e^{-i \zeta / 2} R_{\mathbf{n}}(\zeta)|i\rangle,
$$

where $R_{\mathbf{n}}$ is the rotation operator. $-\zeta / 2$ appears in the output as a global phase which may be incorporated into the algorithm being implemented on the quantum computer [33].

As an example, Fig. 6(a) shows the time evolution of the pumps and Stokes laser light amplitudes in a two-step STIRAP scheme for a single-qubit rotation around the $x$ axis by an angle of $\pi$ with the corresponding time evolution of the DQD populations presented in Fig. 6(b) for the initial state $|i\rangle=0.5|0\rangle+0.866|1\rangle$. We have chosen realistic parameters [34] for the dissipation and decoherence rates given by $\gamma_{\mathrm{SE}}=\gamma_{\mathrm{SD}}=\gamma_{\mathrm{PD}}=2 \mathrm{GHz}$. In this example, the optical control parameters are chosen such that the STIRAP process is completed within $\sim 10 \mathrm{ps}$. According to the adiabatic condition $\Omega_{\max } T \gg 1$, where $\Omega_{\max }$ is the peak value of the Rabi frequency and $T$ is the temporal overlap of the pump and Stokes pulses, the time required to execute a gate is inversely proportional to the pulse Rabi frequencies.

Figure 7 shows the single-particle electron and hole states of each DQD in the two-DQD system presented in Fig. 5. In each DQD, the height of the QDs and their separation should be chosen as a trade-off between good confinement of the electron states and a good overlap with the charged exciton

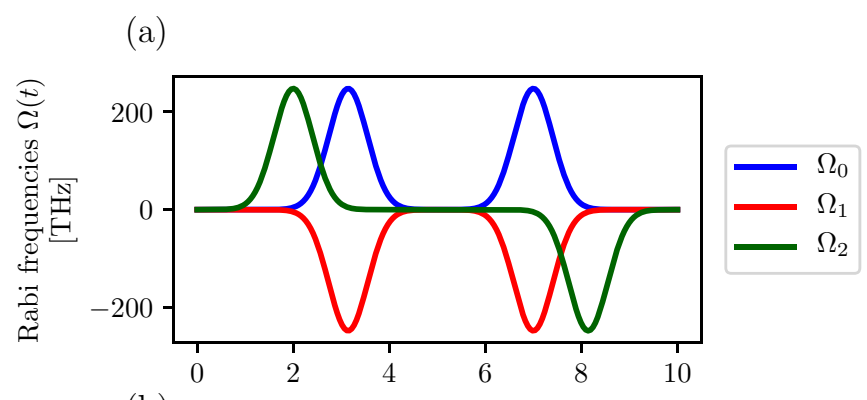

(b)

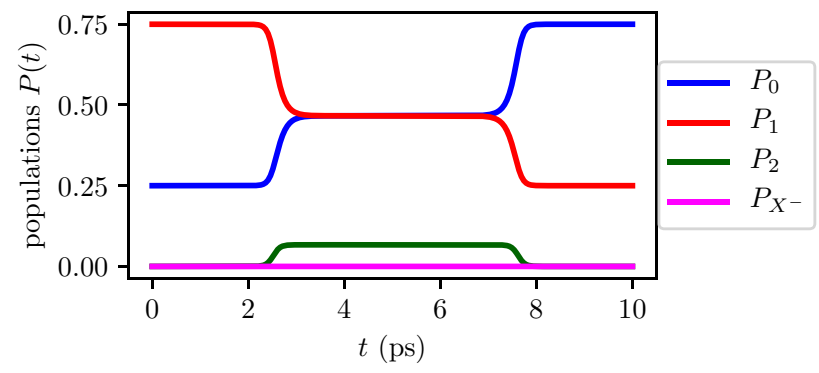

FIG. 6. The time evolution of (a) the laser fields and (b) the populations in a single-qubit NOT operation around the $x$ axis. The initial state is $|i\rangle=0.5|0\rangle+0.866|1\rangle$ and the parameters used in this calculation are $\tau=0.57 \mathrm{ps}, \Delta T=1.14 \mathrm{ps}, \Delta=0, \delta_{1}=\delta_{2}=0$, $\chi=-\pi / 4, \eta=0, \zeta=0(\zeta=\pi)$ for the first (second) STIRAP, $\gamma_{\mathrm{PD}}=\gamma_{\mathrm{SD}}=\gamma_{\mathrm{SE}}=2 \mathrm{GHz}$ and the peak amplitudes of $\Omega_{0}, \Omega_{1}$, and $\Omega_{2}$ are $247 \mathrm{THz}$.

state featuring a hole inside the barrier. By reducing the height of the QDs, the confinement of the single-particle electron states decreases and they diffuse more inside the barrier. This results in a larger overlap with the hole inside the barrier which in turn increases the transition dipole moment. On the other hand, as the height of the QDs decreases below $\sim 2$ $\mathrm{nm}$, the electron states will have a significant overlap with each other and the tunneling probability of the electron states which is not desirable will increase [19]. The same argument also applies to the distance between the QDs. The diameter of the QD should also be chosen as a trade-off between a large dipole moment and sufficient energy level difference between (a)

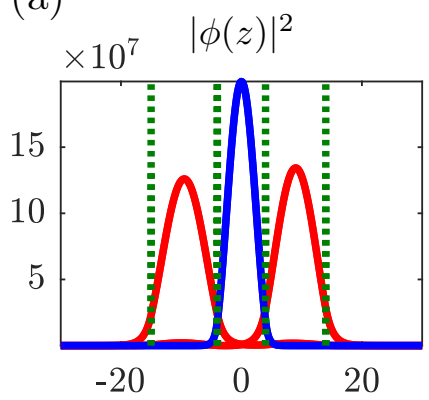

(b)

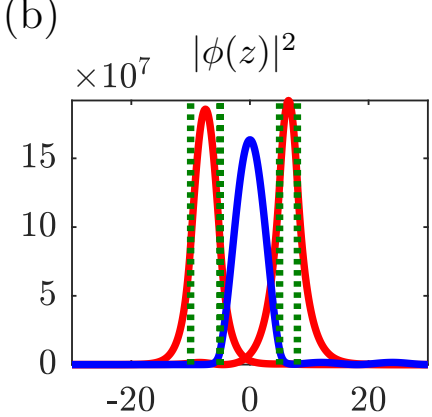

FIG. 7. Normalized single-particle electron (red) and hole (blue) probability densities in the (a) $\mathrm{DQD}_{c}$ and (b) $\mathrm{DQD}_{t}$ of the two-DQD system of Fig. 5. The single-particle energies of $\mathrm{DQD}_{c}$ are $E_{e 1}=$ $31.7 \mathrm{meV}, E_{e 2}=35.1 \mathrm{meV}$, and $E_{h}=6.8 \mathrm{meV}$ and those of $\mathrm{DQD}_{t}$ are $E_{e 1}=68.2 \mathrm{meV}, E_{e 2}=96.6 \mathrm{meV}$, and $E_{h}=5 \mathrm{meV}$. 
(a)

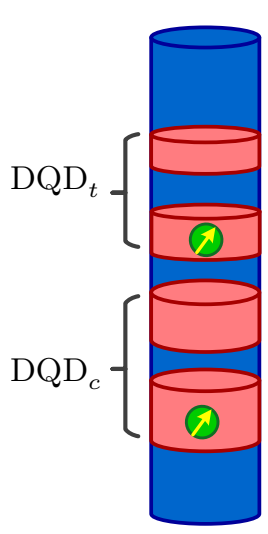

(b)

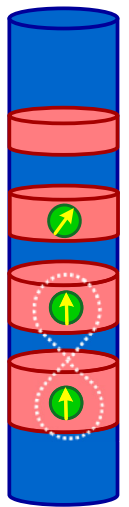

(c)

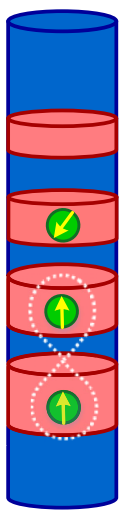

(d)

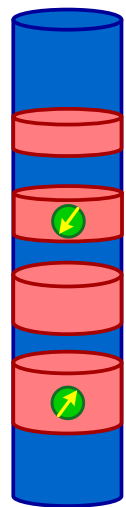

FIG. 8. Illustration of the CNOT gate operation for a nanowire containing two DQDs. (a) The initial state of the structure which includes the control qubit in $\mathrm{DQD}_{c}$ and target qubit in $\mathrm{DQD}_{t}$. (b) The first STIRAP process maps the spin state of the control qubit in $\mathrm{DQD}_{c}$ to the position state. (c) The second and third STIRAP processes act on the target qubit and flips its spin depending on the control qubit state. (d) The fourth STIRAP process returns the control qubit to its initial spin state.

the ground-state charged exciton and the first higher order charged exciton [19,28].

\section{B. Two-qubit gate}

To demonstrate two-qubit operations, we will implement a CNOT gate based on two DQDs in a nanowire as shown in Fig. 5, where the control and target qubits are encoded on $\mathrm{DQD}_{c}$ and $\mathrm{DQD}_{t}$ respectively. The CNOT gate acts by flipping the state of the target qubit if the control qubit is in the state $|1\rangle$ such that, after the CNOT gate operation, the initial two-qubit state $|i\rangle=\alpha_{00}|00\rangle+\alpha_{01}|01\rangle+\alpha_{10}|10\rangle+$ $\alpha_{11}|11\rangle$ is transformed to

$$
|f\rangle=\alpha_{00}|00\rangle+\alpha_{01}|01\rangle+\alpha_{11}|10\rangle+\alpha_{10}|11\rangle .
$$

Implementation of this gate requires a complete population transfer between the $|10\rangle$ and $|11\rangle$ states without perturbing the coefficients of either the $|00\rangle$ or the $|01\rangle$ states.

In the following, we consider two adjacent DQDs with geometrical parameters as listed in the caption of Fig. 5. The initial configuration consists of an electron in $\mathrm{QD}_{1}$ in each DQD of the full system as illustrated in Fig. 8(a). The details of the initialization of this configuration using a sequence of STIRAP processes are presented in Appendix B. The conditional two-qubit CNOT operation then takes place as follows:

Step 1. The first STIRAP process acts on the control qubit in $\mathrm{DQD}_{c}$ and maps the initial spin qubit $\alpha|0\rangle+\beta|1\rangle$ to the position degree of freedom $\alpha|0\rangle+\beta|2\rangle$ as depicted in Fig. 8(b). This is accomplished by choosing the STIRAP parameters $\chi=-\pi / 2, \eta=0$ and $\zeta=0$.

The transfer of an electron from the $|0\rangle$ to the $|2\rangle$ state leads to a new charge distribution of $\mathrm{DQD}_{c}$ which via the Coulomb interaction exerts a potential change on the target qubit in $\mathrm{DQD}_{t}$. This new charge distribution leads to modified $\mathrm{DQD}_{t}$

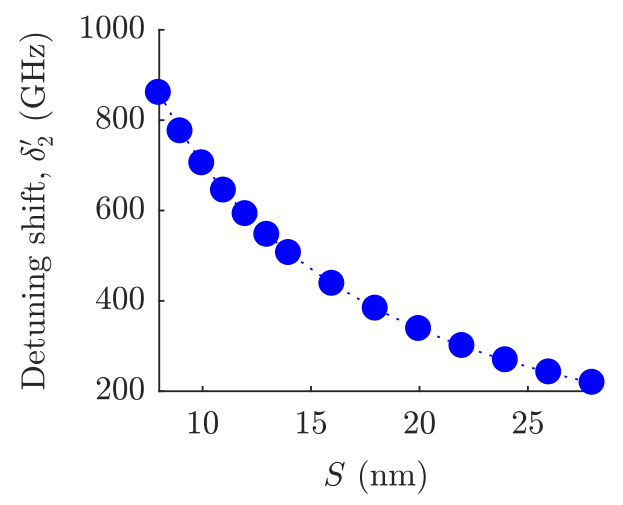

FIG. 9. Shift in two-photon detuning $\delta_{2}^{\prime}$ in $\mathrm{DQD}_{t}$ as a function of the separation, $S$, between $\mathrm{DQD}_{c}$ and $\mathrm{DQD}_{t}$ after step 1 for a control qubit initially in the state $|1\rangle_{c}$.

transition frequencies such that the two-photon detunings are shifted as $\delta_{i} \rightarrow \delta_{i}+\delta_{i}^{\prime}$, where

$$
\delta_{2}^{\prime}=\Delta E_{0}-\Delta E_{2}, \quad \delta_{1}^{\prime}=\Delta E_{0}-\Delta E_{1} .
$$

Here, $\Delta E_{i}=E_{i}^{\prime}-E_{i}(i=0,1,2)$, where $E_{i}$ denotes the energy of the $i$ th electron ground state in $\mathrm{DQD}_{t}$, and the prime sign denotes the new values of the parameters after the first STIRAP. We use the configuration interaction method to calculate the eigenenergies of the two-DQD system including two interacting electrons via Coulomb interaction. In this method, the system Hamiltonian is expanded within the Hilbert space spanned by the lowest energy single-particle electron states. The eigenvalues of this Hamiltonian specify the $E_{i}$ and $E_{i}^{\prime}$ values. The Coulomb-induced shift in $\delta_{2}^{\prime}$ for $\mathrm{DQD}_{t}$ is presented in Fig. 9 as a function of the separation distance $S$ between the two DQDs. We consider a separation $S$ between the DQDs of at least $8 \mathrm{~nm}$ in order to prevent singleparticle tunneling and at the same time to allow for significant Coulomb coupling. Even for fairly large separations $S>8 \mathrm{~nm}$ between the two DQDs, we observe that the strong Coulomb interaction leads to a shift, $\delta_{2}^{\prime}$, of several hundreds of GHz. On the other hand, the shift, $\delta_{1}^{\prime}$, of the two-photon detuning $\delta_{1}$ in $\mathrm{DQD}_{\mathrm{t}}$ is less than $1 \mathrm{GHz}$ for a magnetic field $B$ of a few tesla [35], which is negligible compared to $\delta_{2}^{\prime}$.

Step 2. The second and third STIRAP processes act on the target qubit in $\mathrm{DQD}_{t}$ performing a single-qubit gate with $\chi=$ $-\pi / 4, \eta=0$, and $\zeta=\pi$ as shown in Fig. 8(c). The effect of this single-qubit rotation is strongly dependent on the charge state of the control qubit due to the influence of the Coulomb interaction, which affects $\delta_{2}$.

Step 3. The final STIRAP process with $\chi=-\pi / 2, \eta=0$ and $\zeta=0$ acts on the control qubit in $\mathrm{DQD}_{c}$ by mapping back the position degree of freedom to the spin qubit as shown in Fig. 8(d) thus returning $\mathrm{DQD}_{c}$ to its initial state.

The final result of the STIRAP processes is the rotation of the target qubit alone conditional on the state of the control qubit as required for the two-qubit CNOT operation.

\section{FIDELITY}

We now investigate the performance of our proposed scheme in terms of the fidelity of the single-qubit and 


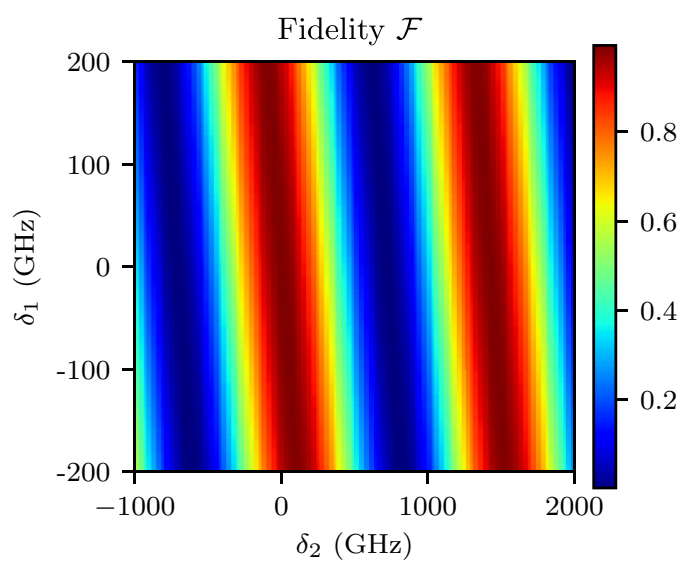

FIG. 10. Fidelity of the NOT gate as a function of two-photon detunings $\delta_{1}$ and $\delta_{2}$ with respect to the ideal NOT gate. The STIRAP parameters in this calculation are as in Fig. 6 and $\gamma_{\mathrm{PD}}=\gamma_{\mathrm{SD}}=$ $\gamma_{\mathrm{SE}}=0$.

two-qubit operations. For scalable quantum information processing, it is essential that our scheme remains robust in the presence of realistic decoherence mechanisms. We discuss our methodology for evaluating the process fidelities and present results of fidelity calculations in the presence of realistic dephasing rates.

\section{A. Single-qubit gate}

We calculate the full single-qubit process fidelities by computing the density matrices for four different initial conditions [36]. If $\rho$ represents the initial density matrix before a gate operation, and $\rho^{\prime}$ represents the final density matrix after the gate operation, then the initial and final density matrices can be related via the process matrix $\chi$ as

$$
\rho^{\prime}=\mathcal{E}(\rho)=\sum_{m n} E_{m} \rho E_{n} \chi_{m n},
$$

where we adopt the basis set $E=\left\{I, \sigma_{x},-i \sigma_{y}, \sigma_{z}\right\}$ and $\sigma_{i}$ are Pauli matrices. By considering initial states as $|0\rangle,|1\rangle,|+\rangle=$ $(|0\rangle+|1\rangle) / \sqrt{2},|-\rangle=(|0\rangle+i|1\rangle) / \sqrt{2}$ and calculating their time evolution using Eq. (4), the process matrix is constructed as

$$
\chi=\Lambda\left[\begin{array}{ll}
\rho_{1}^{\prime} & \rho_{2}^{\prime} \\
\rho_{3}^{\prime} & \rho_{4}^{\prime}
\end{array}\right] \Lambda, \quad \Lambda=\frac{1}{2}\left[\begin{array}{cc}
I & \sigma_{x} \\
\sigma_{x} & -I
\end{array}\right]
$$

where

$$
\begin{aligned}
& \rho_{1}^{\prime}=\mathcal{E}(|0\rangle\langle 0|), \\
& \rho_{4}^{\prime}=\mathcal{E}(|1\rangle\langle 1|), \\
& \rho_{2}^{\prime}=\mathcal{E}(|+\rangle\langle+|)-i \mathcal{E}(|-\rangle\langle-|)-\frac{(1-i)\left(\rho_{1}^{\prime}+\rho_{4}^{\prime}\right)}{2}, \\
& \rho_{3}^{\prime}=\mathcal{E}(|+\rangle\langle+|)+i \mathcal{E}(|-\rangle\langle-|)-\frac{(1+i)\left(\rho_{1}^{\prime}+\rho_{4}^{\prime}\right)}{2} .
\end{aligned}
$$

The process fidelity is then defined as $F=\operatorname{Tr}\left[\chi_{0}\right]$, where $\chi_{0}$ denotes the ideal process matrix.

We initially present the single-qubit NOT gate fidelity in Fig. 10 as a function of the detunings $\delta_{1}$ and $\delta_{2}$ in the absence, $\gamma_{i}=0$, of any dephasing and decay processes. We
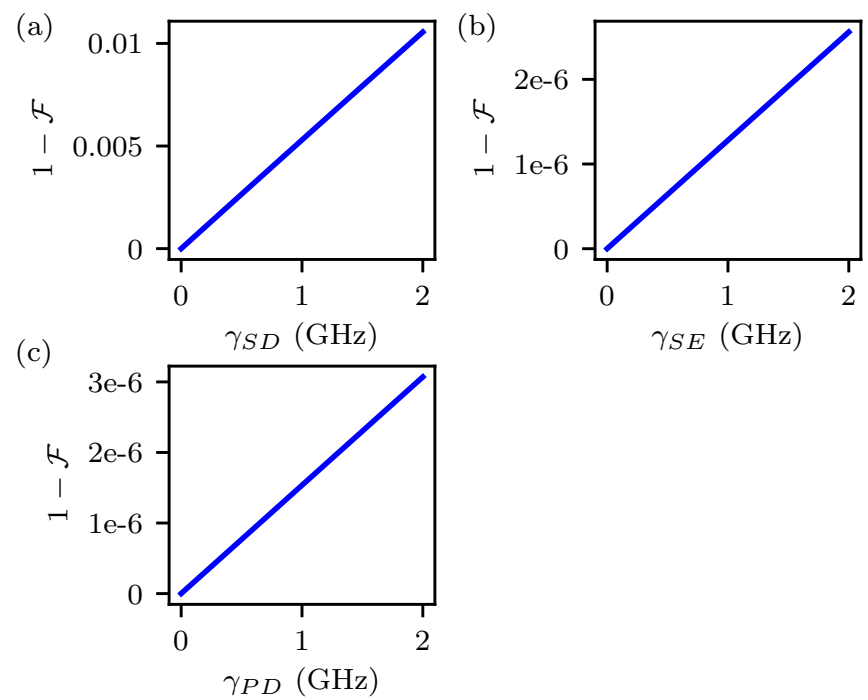

FIG. 11. Infidelity of the NOT-gate as a function of the (a) pure dephasing, (b) spin dephasing, and (c) spontaneous emission. The STIRAP parameters in this calculation are as in Fig. 6.

observe that for $\delta_{1}=\delta_{2}=0$ a high-fidelity NOT operation is achieved. The influence of dephasing rates $\gamma_{\mathrm{SD}}, \gamma_{\mathrm{SE}}$, and $\gamma_{\mathrm{PD}}$ on the NOT-gate fidelity is then presented in Fig. 11. For dephasing rates below $1 \mathrm{GHz}$ representing an upper bound for typical experiments [10,37], a high-fidelity NOT operation is achieved with an infidelity $1-\mathcal{F}$ below 0.005 .

In addition, we observe in Fig. 10 that two-photon detunings of $\delta_{1}=0$ and $\delta_{2}=700 \mathrm{GHz}$ lead to a complete suppression of the NOT operation, and this property provides a means for conditional operation for the two-qubit CNOT gate as discussed in Sec. IV B. The conditional operation of a CNOT gate in a double DQD can be achieved by inducing a Coulomb shift of $700 \mathrm{GHz}$, corresponding to a DQD separation of $10 \mathrm{~nm}$ to hit the interference fringe appearing around $\delta_{2}=$ $700 \mathrm{GHz}$.

\section{B. Two-qubit gate}

To evaluate the two-qubit gate fidelity, we use a two-qubit process matrix [36],

$$
\chi_{2}=\Lambda_{2} \bar{\rho}^{\prime} \Lambda_{2},
$$

where $\Lambda_{2}=\Lambda \otimes \Lambda$ and $\bar{\rho}^{\prime}$ is a block matrix of 16 calculated two-qubit density matrices

$$
\bar{\rho}^{\prime}=P^{T}\left[\begin{array}{cccc}
\rho_{11}^{\prime} & \rho_{12}^{\prime} & \rho_{13}^{\prime} & \rho_{14}^{\prime} \\
\rho_{21}^{\prime} & \rho_{22}^{\prime} & \rho_{23}^{\prime} & \rho_{24}^{\prime} \\
\rho_{31}^{\prime} & \rho_{32}^{\prime} & \rho_{33}^{\prime} & \rho_{34}^{\prime} \\
\rho_{41}^{\prime} & \rho_{42}^{\prime} & \rho_{43}^{\prime} & \rho_{44}^{\prime}
\end{array}\right] P,
$$

where $\rho_{n m}^{\prime}=\mathcal{E}\left(\rho_{n m}\right)$ and $P=I \otimes\left[\left(\rho_{11}+\rho_{23}+\rho_{32}+\rho_{44}\right) \otimes I\right]$ is a permutation matrix. The input two-qubit density matrix operator is the separable operator $\rho_{n m}=\rho_{n}^{c} \otimes \rho_{m}^{t}$, where $c$ and $t$ denote control and target DQDs, respectively. To construct the evolution during the sequence of operations that constitute the CNOT gate in our proposal, we decompose the total evolution as $\mathcal{E}(\rho)=\mathcal{E}_{3}\left(\mathcal{E}_{2}\left(\mathcal{E}_{1}(\rho)\right)\right)$, where each operation $\mathcal{E}_{i}$ corresponds to a step described in Sec. IV B. The first oper- 


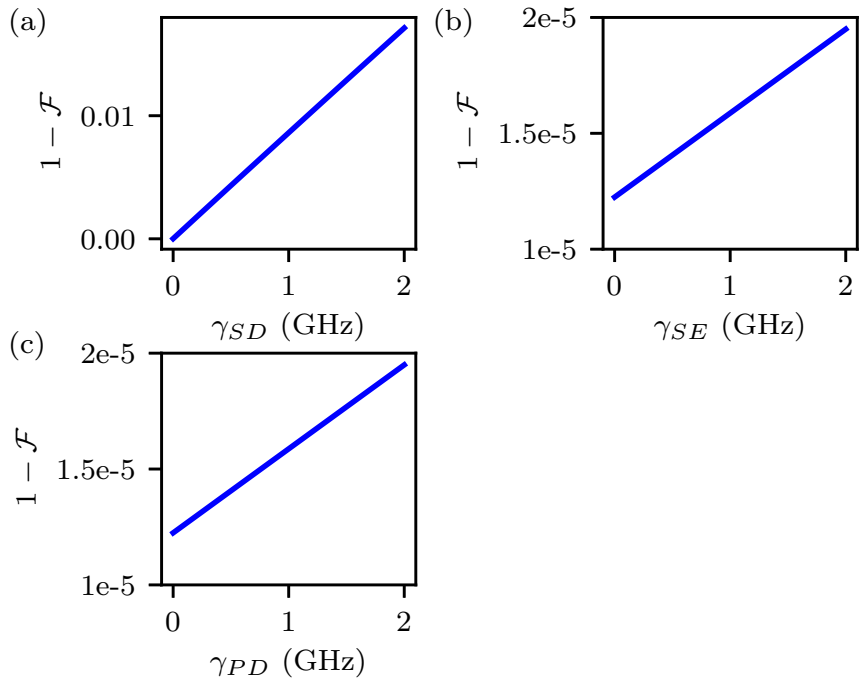

FIG. 12. Infidelity of the two-qubit CNOT gate as a function of the control and target qubits' (a) spin dephasing, (b) spontaneous emission, and (c) pure dephasing rates. The STIRAP parameters of the target qubit are $\chi=-\pi / 4, \eta=0, \tau=0.57 \mathrm{ps}, \Delta T=1.14 \mathrm{ps}$, $\Delta=\delta_{1}=\delta_{2}=0$ and the peak amplitude of $\Omega_{0}, \Omega_{1}$, and $\Omega_{2}$ is 247 THz. The STIRAP parameters of the control qubit are $\chi=-\pi / 2$, $\eta=0, \tau=0.3 \mathrm{ps}, \Delta T=0.6 \mathrm{ps}, \Delta=\delta_{1}=\delta_{2}=0$ and the peak amplitude of $\Omega_{0}, \Omega_{1}$, and $\Omega_{2}$ is $300 \mathrm{THz}$.

ation $\mathcal{E}_{1}$ is simply a STIRAP process with parameters given in Sec. IV B that evolves the control qubit using the master equation, Eq. (4). The second operation $\mathcal{E}_{2}$ performs a NOT operation on the target qubit, where the detuning is subject to a Coulomb shift from the control:

$$
\begin{aligned}
\mathcal{E}_{2}= & (|0\rangle\langle 0|+| 1\rangle\langle 1|)_{c} \otimes \operatorname{NOT}\left(\delta_{2}\right)_{t} \\
& +|2\rangle\left\langle\left. 2\right|_{c} \otimes \operatorname{NOT}\left(\delta_{2}+\delta_{2}^{\prime}\right)_{t},\right.
\end{aligned}
$$

where $\operatorname{NOT}(\delta)$ denotes a single-qubit NOT-operation with two-photon detuning $\delta$. The time evolution under this operation is again calculated using the master equation Eq. (4). The third operation is a STIRAP gate on the control qubit that transfers it back to its initial state. Any errors that have left the target qubit in the state $|2\rangle_{t}$, where the orbital is shifted, will of course lead to errors in this back-transfer process due to an unwanted Coulomb shift on the control qubit. We denote by $\mathrm{T}(\delta)$ the back-transfer STIRAP operation described in step 3 of Sec. IV B with two-photon detuning $\delta$ and write

$$
\begin{aligned}
\mathcal{E}_{3}= & \mathrm{T}\left(\delta_{2}\right)_{c} \otimes(|0\rangle\langle 0|+| 1\rangle\langle 1|)_{t} \\
& +\mathrm{T}\left(\delta_{2}+\delta_{2}^{\prime}\right)_{c} \otimes|2\rangle\left\langle\left. 2\right|_{t},\right.
\end{aligned}
$$

where the evolution under $\mathrm{T}$ is calculated using Eq. (4).

By considering the four combinations of the input states as in Eq. (10) for the control and target qubits, the sixteen elements of $\bar{\rho}^{\prime}$ are determined. The two-qubit gate infidelity $1-\mathcal{F}$ is plotted in Fig. 12 as a function of the dephasing rates $\gamma_{\mathrm{SD}}, \gamma_{\mathrm{SE}}$, and $\gamma_{\mathrm{PD}}$, where we have assumed that the control and target qubits are subject to identical dephasing rates. For dephasing rates $\gamma_{i}$ below $1 \mathrm{GHz}$, we observe that the infidelity $1-\mathcal{F}$ remains below 0.01 .

\section{DISCUSSION}

A relevant merit of the implementation presented in this paper is its robustness against dissipation and decoherence. Of primary concern is the slow fluctuations of the charge environment and the nuclear spins of the host material [4]. Such noise sources influence the system by driving fluctuations of the various energy levels. The timescale of the STIRAP processes should be shorter than the $T_{2}^{*}\left(\simeq \frac{1}{\gamma_{\mathrm{SD}}}\right)$ decoherence time to allow for the application of dynamical decoupling schemes to protect the coherence of the qubit. We chose the STIRAP parameters such that the gating can be implemented on a timescale of a few tens of picoseconds, which is significantly shorter than the typical electron $T_{2}^{*}$ coherence time of a few nanoseconds [38-42]. The robustness of the STIRAP process against the magnitude of the fluctuations depends on the power and duration of the applied laser fields, the overlap time between the two optical pulses, the transition dipole moments and the ground-state Zeeman splitting. We chose these parameters within a realistic range and by solving the master equation including dissipation and decoherence, we predict a two-qubit quantum gate fidelity above 0.99 , thus ensuring robustness of the process against dephasing posed by the environment.

The time required to execute a gate by a conventional STIRAP process considered in this work is inversely proportional to the Rabi frequencies. Thus increasing the input power can facilitate faster operation of the system. However, several strategies have been proposed to speed up the adiabatic passage $[16,43]$, and these can straightforwardly be combined with the proposed scheme to achieve higher gate fidelities in a shorter timescale without increasing the laser power.

We have presented a multiband formalism in Appendix A to evaluate the dipole moments in the STIRAP scheme. Our formalism is compatible with sophisticated methods such as tight-binding or DFT (density functional theory) calculations of the semiconductor bandstructure. However, for design and optimization purposes, numerical simplicity is desired to speed up the calculations, and we have thus chosen to perform the calculations of the dipole moments using a singleband model. Here, we note that our assumption that the $C_{i}$ coefficients in Eqs. (A6) and (A7) are equal is actually not a significant limitation, since the dipole moments for unequal $C_{i}$ coefficients (subject to the requirement that $\Sigma\left|C_{i}\right|^{2}=1$ ) can always be maximized by rotating the polarization of the laser light, and thus we do not expect the dipole moments to be drastically affected by this choice. We stress that our objective is not to calculate exact dipole moments but simply show that the performance of the type II system is superior to that of the type I system. While we acknowledge that more advanced calculation methods may produce different values of the dipole moments, we believe that our conclusion, that the type II system performs significantly better than the type I system, will not be altered by repeating the calculations using such advanced method.

In this work, we used the electron spin states in ZB QDs in a WZ nanowire for the qubit realization. However, by controlling the crystallographic phase of the InP nanowire during the fabrication process it is also possible to fabricate WZ QDs in a ZB nanowire [44]. In this case, the spin of the 
hole confined in the WZ QDs is used as the spin qubit, and a charged exciton state composed of two holes and one electron acts as the intermediate state. Because of the $p$-like symmetry of the hole state at the atomic scale, the hyperfine interaction with the nuclear spins is suppressed, and the hole spin has a longer coherence time in comparison to the electron spin [45]. While the long coherence time of this scheme is attractive, the electron is not a mixed spin state, and thus the charged exciton state cannot couple efficiently to all of the three lowest energy hole states of the DQD as required for single-qubit operations.

\section{CONCLUSION}

We have proposed a novel scheme for all-optical quantum gating based on qubits encoded in ultraclean crystal-phase type II double quantum dots (DQDs) in a nanowire, which are manipulated using stimulated Raman adiabatic passage (STIRAP). The key feature of the scheme is the exploitation of a charged exciton state with a mixed hole which couples with three lowest electron states of the double-quantum-dot in the type II configuration without involving additional external fields. This coupling is essential for high-fidelity qubit rotations by means of the STIRAP process. The two-qubit CNOT operation is performed by using Coulomb coupling to induce a shift of the target DQD transition frequencies leading to a conditional violation of the STIRAP two-photon resonance condition in the target DQD. Using a model accounting for dissipation and environmental noise, we have demonstrated fidelity of the one- and two-qubit gates above 0.99 , thereby showing that for geometrical and dynamical parameters within a realistic range, high-fidelity single- and two-qubit quantum operations can be achieved.

\section{APPENDIX A: CHARGED EXCITON STATE}

In the following, we write the states in the electron picture rather than in the equivalent electron-hole picture such that the many-body states in the DQD structure are approximated as the Slater determinant of a set of single-particle wave functions.

The intermediate charged exciton state $\left|X^{-}\right\rangle$coupling the input state $\alpha|0\rangle+\beta|1\rangle$ to $|2\rangle$ is

$$
\begin{aligned}
\left|X^{-}\right\rangle= & \mathcal{A}\left\{\alpha\left|\uparrow, u_{s}, f_{e 1}\right\rangle+\beta\left|\downarrow, u_{s}, f_{e 1}\right\rangle,\left|\uparrow, u_{s}, f_{e 2}\right\rangle,\right. \\
& \left.\left|\psi_{v 1}\right\rangle, \ldots,\left|\psi_{v(m-1)}\right\rangle,\left|\psi_{v(m+1)}\right\rangle, \ldots,\left|\psi_{v n}\right\rangle\right\} .
\end{aligned}
$$

Here, $|\alpha|^{2}+|\beta|^{2}=1$ and $\mathcal{A}$ is the antisymmetrization operator which we drop henceforth for simplicity. Each state vector in (A1) contains three parts: a spin part, a Bloch function part, and an envelope function part. The conduction band envelope function is denoted by $f_{e}$. Since the conduction band has $s$-like atomic function symmetry, its Bloch function is denoted by $u_{s} .\left|\psi_{v i}\right\rangle$ is the $i$ th valence subband Bloch function; where $i=1, \ldots, n$ with $n \neq m$ such that the valence band is occupied except the $m$ th state. The three upper valence bands are written as a linear combination of Bloch functions $u_{x}, u_{y}, u_{z}$ [46] of the atomic $p$-like symmetry $x, y$, and $z$, respectively. We remark that a different symmetry of the conduction and valence Bloch states can be handled following a similar procedure as presented here. A general valence state $\left|\psi_{v}\right\rangle$ can then be written as

$$
\begin{aligned}
\left|\psi_{v}\right\rangle= & C_{1}\left|\uparrow, u_{x}, f_{h}^{1}\right\rangle+C_{2}\left|\downarrow, u_{x}, f_{h}^{2}\right\rangle+C_{3}\left|\uparrow, u_{y}, f_{h}^{3}\right\rangle \\
& +C_{4}\left|\downarrow, u_{y}, f_{h}^{4}\right\rangle+C_{5}\left|\uparrow, u_{z}, f_{h}^{5}\right\rangle+C_{6}\left|\downarrow, u_{z}, f_{h}^{6}\right\rangle,
\end{aligned}
$$

where $C_{i}$ are the expansion coefficients and $f_{h}^{i}$ with $i=$ $1, \ldots, 6$ are the hole envelope functions which form a complete orthonormal set and can be considered approximately constant over a unit cell. The states $|0\rangle,|1\rangle$, and $|2\rangle$ of the STIRAP scheme in the electron picture are written as

$$
\begin{aligned}
& |0\rangle=\left|\uparrow, u_{s}, f_{e 1}\right\rangle,\left|\psi_{v 1}\right\rangle,\left|\psi_{v 2}\right\rangle, \ldots,\left|\psi_{v n}\right\rangle, \\
& |1\rangle=\left|\downarrow, u_{s}, f_{e 1}\right\rangle,\left|\psi_{v 1}\right\rangle,\left|\psi_{v 2}\right\rangle, \ldots,\left|\psi_{v n}\right\rangle, \\
& |2\rangle=\left|\uparrow, u_{s}, f_{e 2}\right\rangle,\left|\psi_{v 1}\right\rangle,\left|\psi_{v 2}\right\rangle, \ldots,\left|\psi_{v n}\right\rangle,
\end{aligned}
$$

where the valence band is fully occupied by electrons. The transition matrix element between the input state $\alpha|0\rangle+\beta|1\rangle$ and $\left|X^{-}\right\rangle$is now given by

$$
\begin{aligned}
& \left(\alpha\left\langle 0|+\beta\langle 1|) \mathbf{e} \cdot \mathbf{d} \mid X^{-}\right\rangle\right. \\
& \quad=\left\langle\psi_{v m}|\mathbf{e} \cdot \mathbf{d}| \uparrow, u_{s}, f_{e 2}\right\rangle \\
& \quad=C_{1} e_{x} M_{x}\left\langle f_{h}^{1} \mid f_{e 2}\right\rangle+C_{3} e_{y} M_{y}\left\langle f_{h}^{3} \mid f_{e 2}\right\rangle+C_{5} e_{z} M_{z}\left\langle f_{h}^{5} \mid f_{e 2}\right\rangle,
\end{aligned}
$$

where $M_{i}=\left\langle u_{i}\left|\hat{d}_{i}\right| u_{s}\right\rangle ;(i=x, y, z)$ is the bulk matrix element, $\mathbf{e}=\left(e_{x}, e_{y}, e_{z}\right)$ is the unit polarization vector of the laser light and $\mathbf{d}$ is the momentum operator. In the $\mathrm{ZB}$ region, $\left\langle u_{x}\left|\hat{d}_{x}\right| u_{s}\right\rangle=\left\langle u_{y}\left|\hat{d}_{y}\right| u_{s}\right\rangle=\left\langle u_{z}\left|\hat{d}_{z}\right| u_{s}\right\rangle$ and in the WZ region, $\left\langle u_{x}\left|\hat{d}_{x}\right| u_{s}\right\rangle=\left\langle u_{y}\left|\hat{d}_{y}\right| u_{s}\right\rangle \neq\left\langle u_{z}\left|\hat{d}_{z}\right| u_{s}\right\rangle$. Since the electron and hole states spread over the $\mathrm{ZB}$ and the WZ regions, we write

$$
\begin{aligned}
M_{i}\left\langle f_{h} \mid f_{e}\right\rangle= & M_{i}^{\mathrm{ZB}}\left\langle f_{h} \mid f_{e}\right\rangle^{\mathrm{ZB}} \\
& +M_{i}^{\mathrm{WZ}}\left\langle f_{h} \mid f_{e}\right\rangle^{\mathrm{WZ}}, \quad(i=x, y, z) .
\end{aligned}
$$

By considering (A5) and assuming that the light propagates along the $z$ axis, i.e., along the nanowire with a polarization vector normal to the nanowire axis, Eq. (A4) is written as

$$
\begin{aligned}
\left(\alpha\left\langle 0|+\beta\langle 1|) \mathbf{e} \cdot \mathbf{d} \mid X^{-}\right\rangle\right. & \\
= & M^{\mathrm{ZB}}\left\{C_{1} e_{x}\left\langle f_{h}^{1} \mid f_{e 2}\right\rangle+C_{3} e_{y}\left\langle f_{h}^{3} \mid f_{e 2}\right\rangle\right\}^{\mathrm{ZB}} \\
& \left.+M^{\mathrm{WZ}}\left\{C_{1} e_{x}\left\langle f_{h}^{1} \mid f_{e 2}\right\rangle+C_{3} e_{y}\left|f_{h}^{3}\right| f_{e 2}\right\rangle\right\}^{\mathrm{WZ}} .
\end{aligned}
$$

Furthermore, the matrix element of the momentum operator between $|2\rangle$ and $\left|X^{-}\right\rangle$is calculated as

$$
\begin{aligned}
\langle 2| \mathbf{e} & \cdot \mathbf{d}\left|X^{-}\right\rangle \\
= & \left\langle\psi_{v m}|\mathbf{e} \cdot \mathbf{d}| \alpha \uparrow+\beta \downarrow, u_{s}, f_{e 1}\right\rangle \\
= & \left.\alpha\left\{C_{1} e_{x} M_{x}\left\langle f_{h}^{1} \mid f_{e 1}\right\rangle+C_{3} e_{y} M_{y}\left|f_{h}^{3}\right| f_{e 1}\right\rangle\right\} \\
& \left.+\beta\left\{C_{2} e_{x} M_{x}\left\langle f_{h}^{2} \mid f_{e 1}\right\rangle+C_{4} e_{y} M_{y}\left|f_{h}^{4}\right| f_{e 1}\right\rangle\right\} \\
= & M^{\mathrm{ZB}}\left\{\alpha\left(C_{1} e_{x}\left\langle f_{h}^{1} \mid f_{e 1}\right\rangle+C_{3} e_{y}\left\langle f_{h}^{3} \mid f_{e 1}\right\rangle\right)\right. \\
& \left.+\beta\left(C_{2} e_{x}\left\langle f_{h}^{2} \mid f_{e 1}\right\rangle+C_{4} e_{y}\left\langle f_{h}^{4} \mid f_{e 1}\right\rangle\right)\right\}^{\mathrm{ZB}} \\
& +M^{\mathrm{WZ}}\left\{\alpha\left(C_{1} e_{x}\left\langle f_{h}^{1} \mid f_{e 1}\right\rangle+C_{3} e_{y}\left|f_{h}^{3}\right| f_{e 1}\right\rangle\right) \\
& \left.+\beta\left(C_{2} e_{x}\left\langle f_{h}^{2} \mid f_{e 1}\right\rangle+C_{4} e_{y}\left\langle f_{h}^{4} \mid f_{e 1}\right\rangle\right)\right\}^{\mathrm{WZ}} .
\end{aligned}
$$

Equations (A6) and (A7) show, for any value of the $\alpha$ and $\beta$ the charged exciton with a mixed-hole part couples 


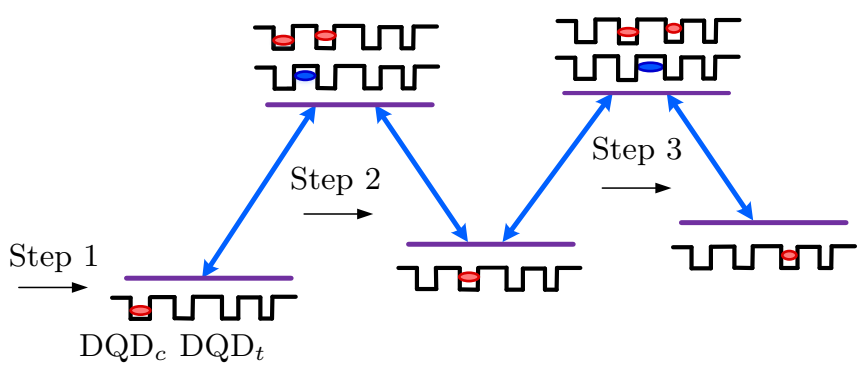

FIG. 13. Scheme for initializing the electrons in a two-DQD. The electron is transferred from $\mathrm{QD}_{1}$ of $\mathrm{DQD}_{c}$ to $\mathrm{QD}_{1}$ of $\mathrm{DQD}_{t}$ using two STIRAP processes.

simultaneously to both the input spin qubits in $\mathrm{QD}_{1}$ as well as to the electron state in $\mathrm{QD}_{2}$. For a charged exciton with a spin-down heavy-hole part for which $C_{1}=C_{3}=0$ then (A6) will be $\left(\alpha\left\langle 0|+\beta\langle 1|) \mathbf{e} \cdot \hat{\mathbf{d}} \mid X^{-}\right\rangle=0\right.$ and for a charged exciton with a spin-up heavy-hole part for which $C_{2}=C_{4}=0$ then (A7) will be $\left\langle 2|\mathbf{e} \cdot \hat{\mathbf{d}}| X^{-}\right\rangle=0$ when $\alpha=0$. In fact, the simultaneous coupling to all ground states is not possible for a charged exciton associated with a pure spin hole part and represents a key asset of the proposed scheme. In addition, Eq. (A7) shows that the dipole moment of the transition $|2\rangle$ to $\left|X^{-}\right\rangle$depends on the input coefficients $\alpha$ and $\beta$. As long as the adiabatic condition $\Omega_{\max } T \gg 1$ is satisfied, this uncertainty does not affect the STIRAP efficiency.

For the numerical calculation in this paper, and since we do not perform a detailed band-structure calculation, we simply assume the envelope functions $f_{h}^{i}$ with $i=1, \ldots, 6$ are the same in the valence subbands with equal expansion coefficients $C_{i}$. With this assumption and taking $M=M^{\mathrm{ZB}}=M^{\mathrm{WZ}}$ and $\alpha=1, \beta=0$, we have calculated the dipole moments $d_{P}=\left\langle 0|\mathbf{e} \cdot \hat{\mathbf{d}}| X^{-}\right\rangle$and $d_{S}=\left\langle 2|\mathbf{e} \cdot \mathbf{d}| X^{-}\right\rangle$presented in the Fig. 3 by using Eqs. (A6) and (A7). We have also assumed a circular polarization light for which $e_{x}=e_{y}=1 / \sqrt{2}$. We point to that the general conclusions are the same for other combinations of $C_{i}$ values. Evaluation of the precise value of these quantities requires a more detailed band-structure calculation, e.g., in the framework of a multiband $k \cdot p$ model or using $a b$ initio atomistic methods [24-26].

\section{APPENDIX B: INITIALIZING ELECTRONS IN DQDS IN A NANOWIRE}

The two-electron configuration illustrated in the Fig. 8(a) can be initialized using the procedure schematically illustrated in Fig. 13.

Step 1. By applying an external electric field in the $z$ direction, an electron from the nearby reservoir is transferred to $\mathrm{QD}_{1}$ of $\mathrm{DQD}_{c}$. Further charging is suppressed because of the Coulomb blockade.

Step 2. This electron is moved to $\mathrm{QD}_{2}$ of $\mathrm{DQD}_{c}$ using one STIRAP operation as shown in Fig. 13.

Step 3. The electron is transferred to the $\mathrm{QD}_{1}$ of $\mathrm{DQD}_{t}$ using another STIRAP operation as shown in Fig. 13.

The $\mathrm{QD}_{1}$ of $\mathrm{DQD}_{c}$ is now empty, and another electron from the reservoir can be transferred to it. The spin of these electrons are initialized by applying a magnetic field.
[1] D. Loss and D. P. DiVincenzo, Phys. Rev. A 57, 120 (1998).

[2] J. Gorman, D. G. Hasko, and D. A. Williams, Phys. Rev. Lett. 95, 090502 (2005).

[3] M. Russ, D. M. Zajac, A. J. Sigillito, F. Borjans, J. M. Taylor, J. R. Petta, and G. Burkard, Phys. Rev. B 97, 085421 (2018).

[4] A. V. Kuhlmann, J. Houel, A. Ludwig, L. Greuter, D. Reuter, A. D. Wieck, M. Poggio, and R. J. Warburton, Nat. Phys. 9, 570 (2013).

[5] H. Bluhm, S. Foletti, I. Neder, M. Rudner, D. Mahalu, V. Umansky, and A. Yacoby, Nat. Phys. 7, 109 (2011).

[6] T. F. Watson, S. G. J. Philips, E. Kawakami, D. R. Ward, P. Scarlino, M. Veldhorst, D. E. Savage, M. G. Lagally, Mark Friesen, S. N. Coppersmith, M. A. Eriksson, and L. M. K. Vandersypen, Nature (London) 555, 633 (2018).

[7] B. E. Kane, Nature (London) 393, 133 (1998).

[8] A. S. Bracker, E. A. Stinaff, D. Gammon, M. E. Ware, J. G. Tischler, A. Shabaev, Al. L. Efros, D. Park, D. Gershoni, V. L. Korenev, and I. A. Merkulov, Phys. Rev. Lett. 94, 047402 (2005).

[9] L. Robledo, J. Elzerman, G. Jundt, M. Atatüre, A. Högele, S. Fält, and A. Imamoglu, Science 320, 772 (2008).

[10] T. S. Koh, S. N. Coppersmith, and M. Friesen, Proc. Natl. Acad. Sci. USA 110, 19695 (2013).

[11] K. D. Petersson, J. R. Petta, H. Lu, and A. C. Gossard, Phys. Rev. Lett. 105, 246804 (2010).
[12] L. A. Orona, J. M. Nichol, S. P. Harvey, C. G. L. Bø ttcher, S. Fallahi, G. C. Gardner, M. J. Manfra, and A. Yacoby, Phys. Rev. B 98, 125404 (2018).

[13] F. Troiani, E. Molinari, and U. Hohenester, Phys. Rev. Lett. 90, 206802 (2003).

[14] N. V. Vitanov, A. A. Rangelov, B. W. Shore, and K. Bergmann, Rev. Mod. Phys. 89, 015006 (2017).

[15] K. Bergmann, H. Theuer, and B. W. Shore, Rev. Mod. Phys. 70, 1003 (1998).

[16] Y. Du, Z. Liang, Y. Li, X. Yue, Q. Lv, W. Huang, X. Chen, H. Yan, and S. Zhu, Nat. Commun. 7, 12479 (2016).

[17] J. J. García-Ripoll and J. I. Cirac, Phys. Rev. Lett. 90, 127902 (2003).

[18] D. P. DiVincenzo, Fortschr. Phys. 48, 771 (2000).

[19] M. Taherkhani, M. Willatzen, J. Mørk, N. Gregersen, and D. P. S. McCutcheon, Phys. Rev. B 96, 125408 (2017).

[20] C. Fasth, A. Fuhrer, M. T. Björk, and L. Samuelson, Nano Lett. 5, 1487 (2005).

[21] S. M. Frolov, S. R. Plissard, S. Nadj-Perge, L. P. Kouwenhoven, and E. P. A. M. Bakkers, MRS Bull. 38, 809 (2013).

[22] Y. Wang, X. Zhang, T. A. Corcovilos, A. Kumar, and D. S. Weiss, Phys. Rev. Lett. 115, 043003 (2015).

[23] I. E. Linington and N. V. Vitanov, Phys. Rev. A 77, 010302(R) (2008). 
[24] M. B. Bavinck, K. D. Jöns, M. Zieliski, G. Patriarche, J. C. Harmand, N. Akopian, and V. Zwiller, Nano Lett. 16, 1081 (2016).

[25] O. Marquardt, M. Ramsteiner, P. Corfdir, L. Geelhaar, and O. Brandt, Phys. Rev. B 95, 245309 (2017).

[26] P. E. F. Junior and G. M. Sipahi, arXiv:1202.2804.

[27] P. E. F. Junior and G. M. Sipahi, J. Appl. Phys. 112, 103716 (2012).

[28] U. Bockelmann and G. Bastard, Phys. Rev. B 42, 8947 (1990).

[29] I. Vurgaftman, J. R. Meyer, and L. R. Ram-Mohan, J. Appl. Phys. 89, 5815 (2001).

[30] Z. Kis and F. Renzoni, Phys. Rev. A 65, 032318 (2002).

[31] P. Chen, C. Piermarocchi, L. J. Sham, D. Gammon, and D. G. Steel, Phys. Rev. B 69, 075320 (2004).

[32] G. S. Agarwal, Quantum Optics, 1st ed. (Cambridge University Press, Cambridge, 2013).

[33] Z. Kis and E. Paspalakis, Phys. Rev B. 69, 024510 (2004).

[34] A. Bechtold, D. Rauch, F. Li, T. Simmet, P.-L. Ardelt, A. Regler, K. Müller, N. A. Sinitsyn, and J. J. Finley, Nat. Phys. 11, 1005 (2015).

[35] M. Trif, V. N. Golovach, and D. Loss, Phys. Rev. B 75, 085307 (2007).

[36] I. L. Chuang and M. A. Nielsen, J. Mod. Optics 44, 2455 (1997).
[37] T. Hayashi, T. Fujisawa, H. D. Cheong, Y. H. Jeong, and Y. Hirayama, Phys. Rev. Lett. 91, 226804 (2003).

[38] D. Press, K. De Greve, P. L. McMahon, T. D. Ladd, B. Friess, C. Schneider, M. Kamp, S. Höfling, A. Forchel, and Y. Yamamoto, Nat. Photon. 4, 367 (2010).

[39] E. V. Denning, J. Iles-Smith, D. P. S. McCutcheon, and J. Mork, Phys. Rev. A 96, 062329 (2017).

[40] G. Éthier-Majcher, D. Gangloff, R. Stockill, E. Clarke, M. Hugues, C. Le Gall, and M. Atatüre, Phys. Rev. Lett. 119, 130503 (2017).

[41] X. Xu, B. Sun, P. R. Berman, D. G. Steel, A. S. Bracker, D. Gammon, and L. J. Sham, Nat. Phys. 4, 692 (2008).

[42] A. Greilich, D. R. Yakolev, A. Shabaev, A. L. Efros, I. A. Yugova, R. Oulton, V. Stavarache, D. Reuter, A. Wieck, and M. Bayer, Science 313, 341 (2006).

[43] B. B. Zhou, A. Baksic, H. Ribeiro, C. G. Yale, F. J. Heremans, P. C. Jerger, A. Auer, G. Burkard, A. A. Clerk, and D. D. Awschalom, Nat. Phys. 13, 330 (2017).

[44] X. Yan, X. Zhang, J. Li, Y. Wu, and X. Ren, Appl. Phys. Lett. 107, 023101 (2015).

[45] D. Brunner, B. D. Gerardot, P. A. Dalgarno1, G. Wüst, K. Karrai, N. G. Stoltz, P. M. Petroff, and R. J. Warburton, Science 325, 70 (2009).

[46] E. O. Kane, J. Phys. Chem. Solids 1, 249 (1957). 